\title{
Development of an Interactive Virtual Reality for Medical Skills Training supervised by Artificial Neural Network
}

\author{
Shabnam Sadeghi Esfahlani ${ }^{1}$, Viktor Izsof ${ }^{1}$, Sabrina Minter ${ }^{2}$, Ali Kordzadeh ${ }^{3}$, Hassan Shirvani ${ }^{1}$ \\ ${ }^{1}$ Faculty of Science and Technology \\ ${ }^{2}$ Faculty of Arts, Law and Social Sciences \\ ${ }^{3}$ Faculty of Medical Sciences \\ Anglia Ruskin University, Cambridge, UK \\ Email: shabnam.sadeghi-esfahlani@anglia.ac.uk \\ viktor.izsof@student.anglia.ac.uk \\ billy@flamrock.com \\ ali.kordzadeh@pgr.anglia.ac.uk \\ hassan.shirvani@anglia.ac.uk
}

\begin{abstract}
The simulation of medical procedures through virtual reality (VR) system offers an opportunity to train medical students, paramedics and doctors across a range of specialists in a safe (risk-free), realistic and repeatable environment. It eliminates harmful consequences to patients and offers infinitely repeatable procedures. In this study, we developed an application of a "single Interrupted Suturing skill" that is used daily for various fields of surgery which is supervised by artificial intelligence (AI) algorithm. The automated graphical feedback and assessment procedures were simulated within the VR environment. The VR technologies for medical/clinical training has the potential to reduce service delivery costs significantly. The evaluation of the effectiveness and validity of the proposed system will be the focus of future research.
\end{abstract}

Index Terms-Virtual Reality (VR), Serious Games, Single Interrupted Suturing, Medical Training, Artificial Intelligence (AI).

\section{INTRODUCTION}

In the last decades, serious games and virtual reality (VR) applications have received much attention for education, training purposes and to enrich the learning process. VR system could provide a realistic environment for Medical training/applications that offers the recreation of the real circumstances/training of the procedures. Furthermore, the development of haptic devices enhanced the VR experience in that the user can see, touch, hear and feel the virtual objects [1]. In medicine, the presence of interactive and $3 \mathrm{D}$ visualization offers an immersive simulation [2] which provides the possibility of training without risks. Additionally, these applications avoid the damage of materials and also can present models and situations that are difficult/impossible to be trained in real conditions. The technology encompasses 3D modeling, simulation, associated display and inputs (devices) that lend themselves well to the nature of surgery [2]. The application possibilities of VR technology are innumerable, and many industries have already found areas to use this technology or are actively exploring its possibilities [3]. While specific steps were taken to incorporate VR-based training environments for medical students and novice clinicians [4], many still use traditional methods. In recent times, the growing awareness of the need for patient safety has led to the traditional approach to be considered insufficient [5], [6]. Various studies have confirmed the effectiveness of utilizing VR technology for training and education purposes [7], [8], [9], [10], [11], [12]. According to [4], VR medical training system could have a scientific quality if it meets specific criteria including; feedback, deliberate practice, outcome measurement, simulation fidelity, skill acquisition and maintenance, mastery learning, transfer to practice, instructor training, and educational and professional context. Many scientific experiments have examined the potential benefits of utilizing immersive and interactive VR environments for various sections of medical education, explicitly achieving surgical competence and dexterity without the risks of the traditional methods or the potential mistakes caused by lack of experience [13]. The project aims to design and develop an application formed of clinical training scenarios in VR environments to train the medical student. It would provide them with the opportunity to repeatedly practice appropriate surgical procedures while eliminating harmful consequences to patients. Our case study focuses on developing a simple suturing practice through an immersive environment and AI algorithm. The feedback provided through the AI system that collects the data from the player and compares it with the database to assess the player's performance accordingly. We have developed the prototype based on the creation of knots in a set order, a unique approach to detecting circular motion and the direction of the circular motion. The environment detects the relative movement of the users hands through the HTC Vive controllers. The 3D environment was simulated through the head-mounted display. Our fully interactive, scenariobased environment could enable medical students to practice 
surgical exercises and have access to a risk-free, fast and infinitely repeatable method of training.

\section{A. HTC Vive Headset}

The HTC Vive headset utilizes room scale tracking technology, enables the user to move in a 3D environment and use motion-tracked handheld controllers to communicate with the environment. The headset has a refresh rate of $90 \mathrm{~Hz}$ with $110^{\circ}$ degree field of view. The VR device employs two OLED panels (one per eye) each having a display resolution of $1080 \times 1200$. It has safety features with a front-facing camera which entitles the user to observe their surroundings without removing their headset. The Vive controllers have multiple input methods including a trackpad, grip buttons, and a dual-stage trigger. Beyond the ring of the controller is twenty-four infrared sensors that detect the base stations to determine the location of the controller ${ }^{1}$. Figure 1 displays the HTC Vive headset, controllers and the base station. The SteamVR tracking system is used to track the controller location to a fraction of a millimeter $(\mathrm{mm})$, with update rates; $[250-1000] \mathrm{Hz}$. Lighthouse tracking system (base stations) are two black boxes that create a $360^{\circ}$ virtual space up to $15 \times 15$ foot radius $^{2}$.

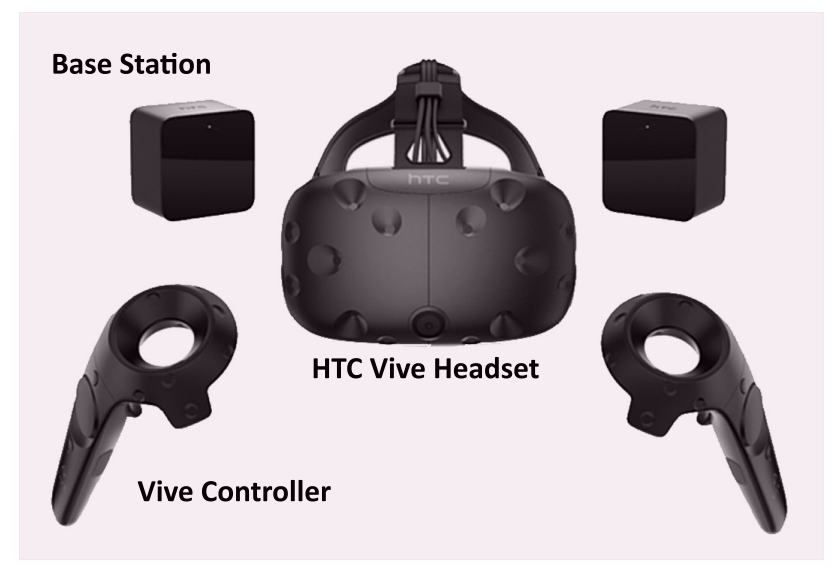

Fig. 1. HTC Vive head-mounted display with two wireless hand held controllers and two base stations

\section{Design And Methodology}

\section{A. Artificial Neural Network \& Virtual Reality}

One of the keys to a compelling virtual world is for the user to be able to suspend disbelief. That is, the user must be able to imagine that the world represented is real, without being bounced off this belief by the world's behavior [14]. Virtual reality is a collection of technologies that allow people to interact efficiently with $3 \mathrm{D}$ computerized databases in real time using their natural senses and skills. We aim at developing a highly immersive VR environment to enable players to

\footnotetext{
${ }^{1}$ https://www.vrheads.com/exposing-magic-behind-htc-vive-controller ${ }^{2}$ https://partner.steamgames.com/vrlicensin
}

function as they do in real-world practice. It incorporates $3 \mathrm{D}$ modeling, simulation, associated display, input devices and haptic effects which lend themselves well to the nature of the surgery. The artificial intelligence (AI) algorithm supervises the player throughout the training process. The real-time feedback was provided through the graphical and heads-up display (HUD) as the player follows the steps in the game. Adaptive learning algorithms and AI could perform effectively and independently in a dynamic environment. They manipulate several inputs to categorize them into the outputs. The artificial neural network (ANN) provide real-time feedback to the player in a dynamic VR training environment. The collected data are used to train and regulate the behavior of the algorithm (agent). It utilizes the diverse types of input data in the context of previous training to classify inputs into outputs. The system creates a closed-loop of real behaviors inserted into artificial agents [15]. Artificial Neural Networks (ANNs) are

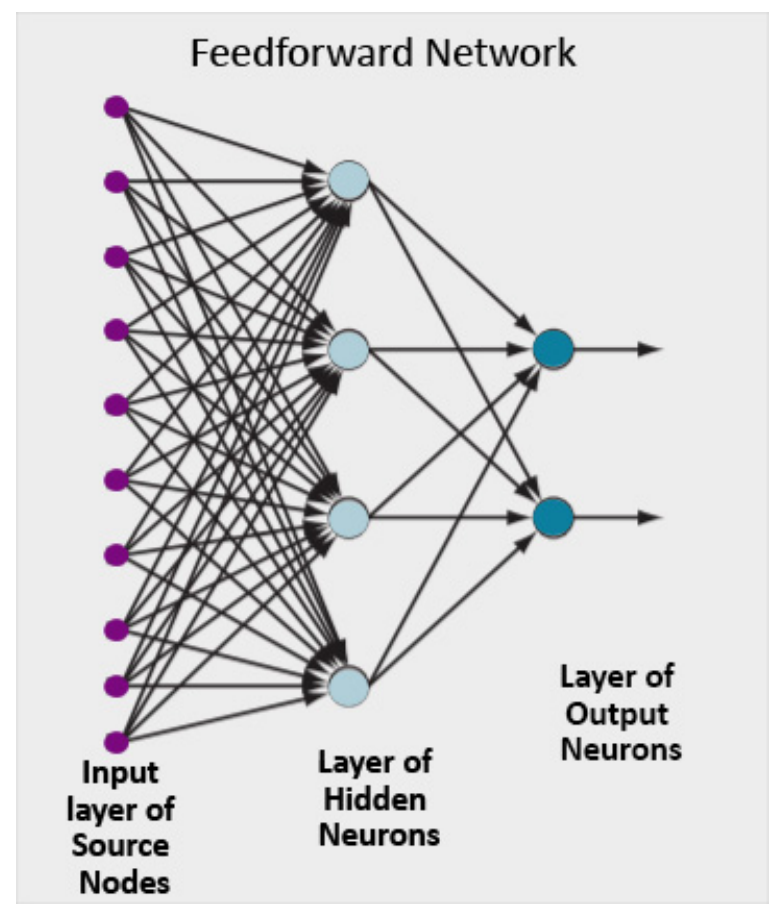

Fig. 2. The NN algorithm's structure with input, hidden and output layers [16].

the mathematical algorithms, generated by computers. ANNs learn from standard data and capture the knowledge contained in the data [17] and can detect complex nonlinear relationships with in data [18], [19]. Figure 2 illustrates the NN algorithm with its various layers.

Figure 3 illustrates the structure of a neural networks which could be written in the mathematical terms as follows:

$$
\begin{array}{r}
u_{k}=\sum_{i=1}^{m} \omega_{k i} p_{i} \\
y_{k}=\varphi\left(u_{k}+b_{k}\right) \\
\nu_{k}=u_{k}+b_{k}
\end{array}
$$

In that the input signals $p_{i}$; are multiplied by the respective synaptic weights of neuron $k, w_{k i} . u_{k}$ is the linear combiner 


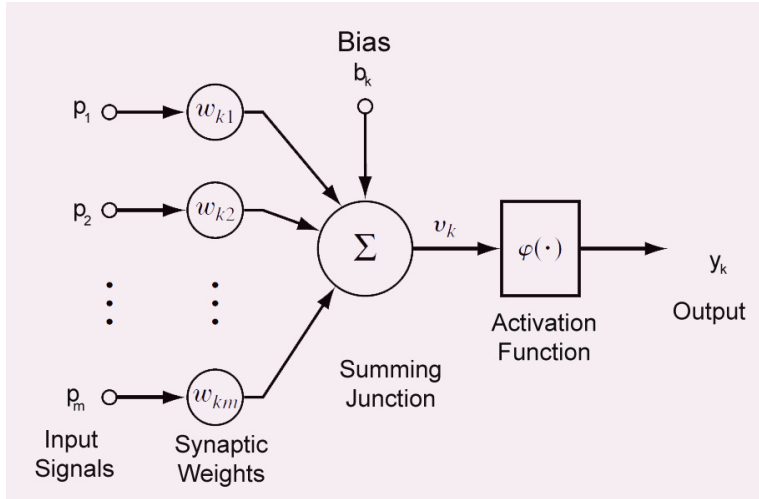

Fig. 3. Nonlinear model of a neuron with labeled $k$.

output due to the input signals; $b_{k}$ is the bias; $\varphi($.$) is the$ activation function (Log-Sigmoid), and $y_{k}$ is the output signal of the neuron. The Log-Sigmoid is the argument of the differentiable transfer function which is used in the hidden layers of multilayer back-propagation network. Log-Sigmoid was used for pattern recognition problems that take the input $[-\infty,+\infty]$, and squashes the output into the range of $[0,1]$. Classification algorithm, the feed-forward back-propagation neural network is a curve fitting in that units are connected based on their strength/weight. The weights on these connections encode the knowledge of a network. Feed-forward neural networks (FFNN) often have one or more hidden layers of Sigmoid neurons followed by an output layer of linear neurons.

\section{B. Interrupted Surgeons Suturing}

Game-Based learning/training has been used to address specific real-world problematic areas employing serious games with pre-defined learning outcomes [20]. Figure 4 and Figure 5 show the game scenario developed for interrupted surgeon suturing/knot training. Figure 4-a,b illustrate the surgery room and the body with an open wound. Figure 4-c displays the stitched wound with the material and its mesh in Unity. Figure 5 and Figure 6 show the scene while the player interacts with the virtual objects via the controllers and follows the suturing process. To simulate a realistic VR scenario the following steps were followed which is based on the suturing video training based on a mat used in traditional training.

The classification of the main milestones of the scenario is as follows:

- Picking up the tools

- Inserting the needle into the virtual dummy

- Guiding the needle through to the exit position

- Grabbing the needle on the other end

- Pulling the needle out

- Putting the needle to the side

- Using the forceps grabbing one of the threads

- Movement of the opposite hand around the forceps to create the Knot

- Pulling the two hands apart to tie the suture/knot above the wound
- Repeat the suture/knot tying process the required times for the Interrupted Surgeon's Suturing

The identification and design of the tools the user would use during the scenario are;

- Surgeons needle

- Forceps to hold the needle and act as the central point for creating the knots after insertion

- The threaded ball used to showcase the circular motion around the forceps to create the correct knots

- The wound that would act as the training area for the sewing itself

Achieving all of these points would create a one to one representation of the real-world process of creating the simple interrupted surgeon's suture in VR and using the objective feedback system achieve a more streamlined process with specific information being fed back to the user about their exercise in the virtual training scenario. Having the immediate feedback would allow the user to improve their performance in mastering this scenario faster than in the real world as they could continue practicing without the need to resupply any material that would have been used up in the real world during the scenario. Figure 5 illustrates the screenshot of the game scenario and the procedure.

\section{RESULTS \& DISCUSSION}

\section{A. Results}

It was developed as an alternative training method for traditional suturing practice. The traditional suturing used surgical kit; mat and tools to practice suturing. The threat of deformation and stereoscopic visualization also need to be entirely implemented. The system was developed to record the player's activity and performance in the hard drive. Figure 7 shows the data collected via the controllers as the player follows the steps. Methods for collision detection, deformation and stereoscopic visualization also need to be entirely implemented.

The application of this technology to surgical training is evident. Surgical training is expensive, and the pressures from shortened training programmes and reduced working hours for trainees demand that an increasing proportion of the surgical expertise of trainees has to be gained outside the operating theatre. The crucial factor that will determine the uptake of virtual reality technology by surgeons will be the demonstration that virtual reality is capable of delivering reliable and valid training and assessment systems. Recent evidence suggests that this is the case. simple Interrupted surgeons knot scenario was selected and as such the goal was to design and develop the main features and functionality.

The result of a double-blinded study demonstrated that VR training transfers technical skills to the operating room environment with significantly improved performance over non-VR-trained residents [21]. A significant complication that developers continuously attempt to successfully and consistently address is the creation and maintenance of an immersed state while not breaking the illusion of presence [3] and [22]. 


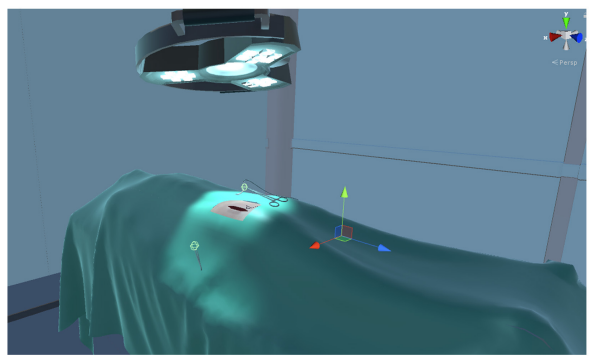

(a)

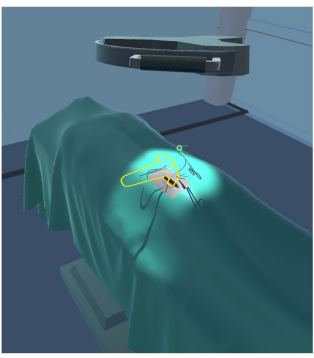

(b)

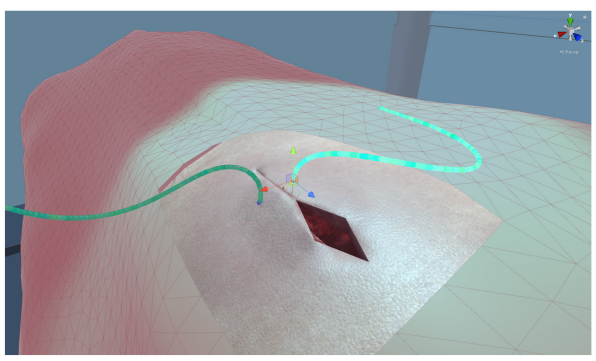

(c)

Fig. 4. VR scenario developed for the training.

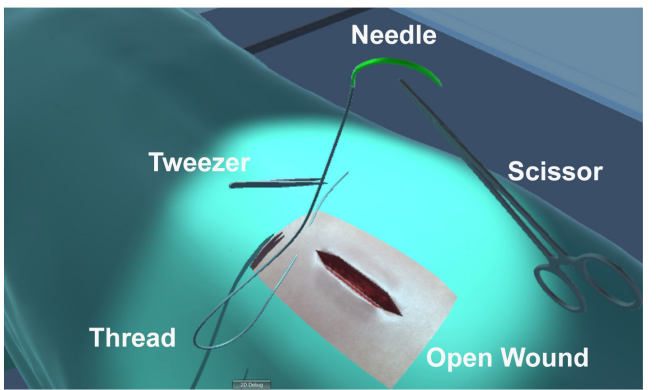

(a)

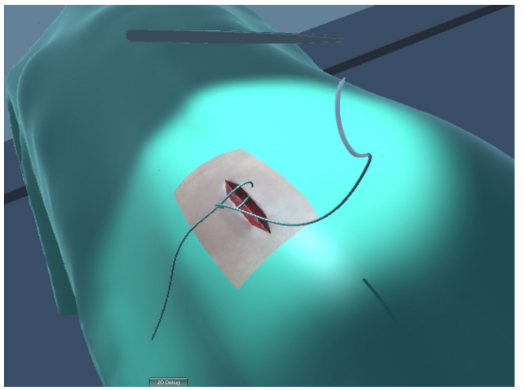

(c)

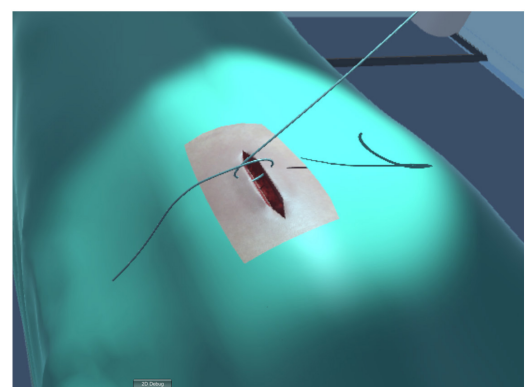

(b)

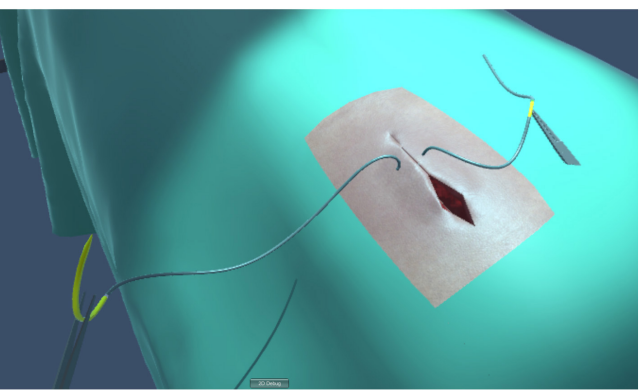

(d)

Fig. 5. Virtual reality game scenario with the interrupted surgeon suturing.

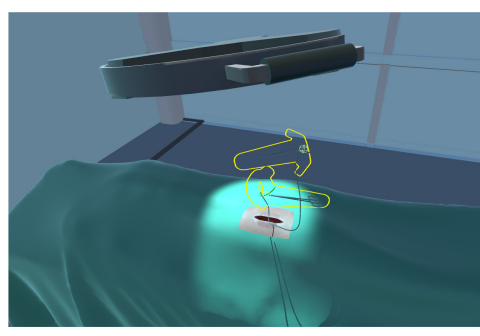

(a)

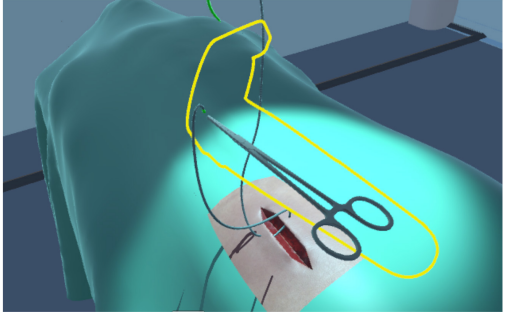

(b)

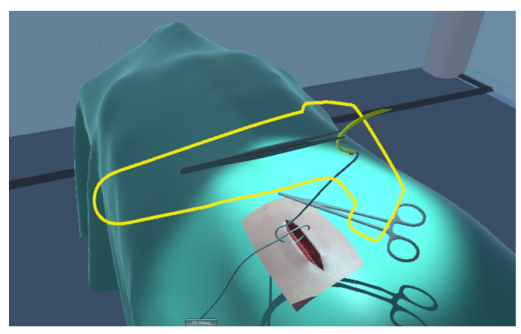

(c)

Fig. 6. Screen shots of the suturing process and interaction with virtual tweezer, needle and scissor through HTC controllers. 


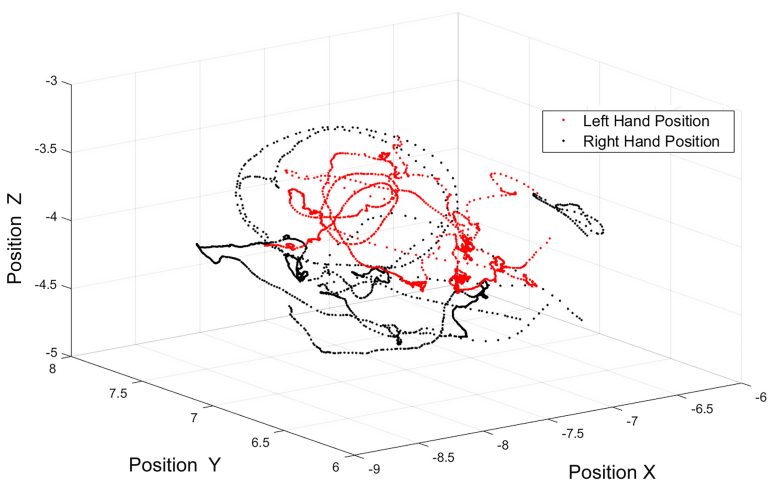

(a)

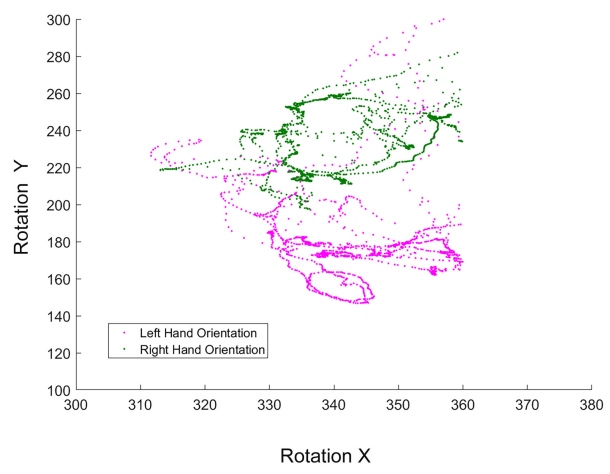

(b)

Fig. 7. Scatter plot of the hands position and orientation while interacting with the VR scenario.

\section{Discussions}

\section{A. Discussion \& Conclusion}

In this study, a basic surgery suture training was developed in a VR environment using HTC Vive. It aimed to develop an interactive VR environment that could provide a better experience and skills in real-time practice compared to the traditional methods. The scenarios, models, and haptic feedback from the video-game controllers were implemented to create an immersive environment and interact with a virtual tweezer, scissor suturing thread and needle and receive haptic feedback like a real-world physical exercise. The realistic material effects were simulated in the Unity game engine. The result has shown that the VR system offers a safe environment for training while the user could learn from the errors through the visual feedback and AI algorithm. It provided an environment that could enable the user to interact and practice as many time as they want in a safe environment while the actions were assessed with the AI algorithm. The AI system collects the data from the player and assesses the performance accordingly. Hands vibration, position, and orientation were collected to assess the performance quality, hands orientation, procedures, and timing. Real-time feedback enabled the player to correct the mistakes which offer a standardized experience for the users.

This research has some limitations which will be addressed in our future work. The forthcoming study will look into comparing the VR and traditional methods, considering a sufficient sample size for validation and statistical tests.

\section{REFERENCES}

[1] T. Pletcher, K. Bier, and D. von Lubitz, “An immersive virtual reality platform for medical education: Introduction to the medical readiness trainer," in hicss, p. 5025, IEEE, 2000.

[2] A. Al-Khlaifah and D. Roberts, "A survey of modeling approaches for medical simulators," International Journal on Disability and Human Development, vol. 4, no. 3, pp. 153-160, 2005.

[3] J. Jerald, The VR book: Human-centered design for virtual reality. Morgan \& Claypool, 2015.

[4] W. C. McGaghie, S. B. Issenberg, E. R. Petrusa, and R. J. Scalese, "A critical review of simulation-based medical education research: 20032009," Medical education, vol. 44, no. 1, pp. 50-63, 2010.
[5] H. R. Patel and B. P. Patel, "Virtual reality surgical simulation in training," Expert review of anticancer therapy, vol. 12, no. 4, pp. 417420, 2012.

[6] C. M. Walsh, M. E. Sherlock, S. C. Ling, and H. Carnahan, "Virtual reality simulation training for health professions trainees in gastrointestinal endoscopy," The Cochrane Library, 2012.

[7] B. Dalgarno and M. J. Lee, "What are the learning affordances of 3d virtual environments?," British Journal of Educational Technology, vol. 41, no. 1, pp. 10-32, 2010.

[8] F. Aïm, G. Lonjon, D. Hannouche, and R. Nizard, "Effectiveness of virtual reality training in orthopaedic surgery," Arthroscopy: The Journal of Arthroscopic \& Related Surgery, vol. 32, no. 1, pp. 224-232, 2016.

[9] A. Bernardo, "Virtual reality and simulation in neurosurgical training," World neurosurgery, vol. 106, pp. 1015-1029, 2017.

[10] A. Menin, R. Torchelsen, and L. Nedel, "An analysis of vr technology used in immersive simulations with a serious game perspective," IEEE computer graphics and applications, vol. 38, no. 2, pp. 57-73, 2018.

[11] C. Fertleman, P. Aubugeau-Williams, C. Sher, A.-N. Lim, S. Lumley, S. Delacroix, and X. Pan, "A discussion of virtual reality as a new tool for training healthcare professionals," Frontiers in public health, vol. 6, p. 44, 2018.

[12] T. Mazur, T. R. Mansour, L. Mugge, and A. Medkour, "Virtual reality based simulators for cranial tumor surgery: A systematic review," World neurosurgery, 2017.

[13] J. Bartlett, J. Lawrence, M. Stewart, N. Nakano, and V. Khanduja, "Does virtual reality simulation have a role in training trauma and orthopaedic surgeons?," Bone Joint J, vol. 100, no. 5, pp. 559-565, 2018.

[14] J. Bates, "Virtual reality, art, and entertainment," Presence: Teleoperators \& Virtual Environments, vol. 1, no. 1, pp. 133-138, 1992.

[15] P. Cipresso and G. Riva, "Virtual reality for artificial intelligence: human-centered simulation for social science," in Annual Review of Cybertherapy and Telemedicine 2015: Virtual Reality in Healthcare: Medical Simulation and Experiential Interface, IOS Press, 2015.

[16] S. S. Haykin, S. S. Haykin, S. S. Haykin, and S. S. Haykin, Neural networks and learning machines, vol. 3. Pearson Upper Saddle River, 2009.

[17] H. Beale and Demuth, "Neural network toolbox for use with matlab," 2018.

[18] F. Amato, A. López, E. M. Peña-Méndez, P. Vaňhara, A. Hampl, and J. Havel, "Artificial neural networks in medical diagnosis," 2013.

[19] V. N. Vapnik, "An overview of statistical learning theory," IEEE transactions on neural networks, vol. 10, no. 5, pp. 988-999, 1999.

[20] F. Bellotti, B. Kapralos, K. Lee, P. Moreno-Ger, and R. Berta, "Assessment in and of serious games: an overview," Advances in HumanComputer Interaction, vol. 2013, p. 1, 2013.

[21] N. E. Seymour, A. G. Gallagher, S. A. Roman, M. K. Obrien, V. K. Bansal, D. K. Andersen, and R. M. Satava, "Virtual reality training improves operating room performance: results of a randomized, doubleblinded study," Annals of surgery, vol. 236, no. 4, p. 458, 2002.

[22] J. Psotka, "Immersive training systems: Virtual reality and education and training," Instructional science, vol. 23, no. 5-6, pp. 405-431, 1995. 